\title{
RAZONAR EN LO JURÍDICO Y EN LO MORAL, DOS RAZONES, DIFERENTES RACIONALIDADES
}

\author{
Reasoning in the legal and moral domain. Two reasons, different \\ rationalities
}

Jorge Arturo Cerdio Herrán*

\begin{abstract}
Resumen: El artículo adopta como objeto temático el razonamiento jurídico. Sostiene la tesis de que la distinción entre razón teórica y razón práctica es clave para entender la naturaleza del razonamiento jurídico. La tesis se argumenta a partir de la distinción entre razón teórica y razón práctica. Por un lado, el razonamiento teórico tiene relación directa con el conocimiento acerca del mundo. De otro, el razonamiento práctico se ocupa acerca de decidir el curso de acción a elegir en un caso concreto. Aunque relacionadas, las racionalidades son regidas por diferentes criterios de evaluación. En segundo término se caracteriza al razonamiento práctico moral dentro del dominio exclusivo de la razón práctica y se analiza su estructura y criterios de evaluación. Finalmente se distingue el razonamiento jurídico de la moral en tanto el primero tiene exigencias de la razón teórica (conocimiento de hechos y conocimiento de normas) y de la razón práctica (qué hacer en un caso particular). Las diferentes exigencias de razón permiten advertir, también, que ciertos razonamientos jurídicos son pasibles de ciertos sentidos de racionalidad que no son aplicables al razonamiento moral.
\end{abstract}

Palabras clave: razonamiento jurídico - razón teórica - razón práctica - razonamiento práctico moral.

Abstract: The article takes as its thematic object legal reasoning. It proposes that the distinction between theoretical reason and practical reason is the key to better understand the nature of legal reasoning. The thesis is argued from the distinction between theoretical reason and practical reason. On the one hand, theoretical reasoning is directly related to gaining knowledge about the world. On the other hand, practical reasoning is concerned about deciding the best course of action in a particular situation. Although related, the two rationales are governed by different evaluation criteria. The article also analyzes moral practical reasoning as a reasoning that belongs exclusively to the domain of practical reason. It's structure and evaluation criteria are discussed. Finally, the paper distinguishes moral from legal reasoning in that the legal reasoning has requirements of the theoretical reason (knowledge of facts and knowledge of rules) and from the practical reason

\footnotetext{
* Abogado. Doctor en Derecho por la Universidad de Buenos Aires, Argentina. Profesor Numerario de Tiempo Completo del Departamento Académico de Derecho del Instituto Tecnológico Autónomo de México. Correo electrónico: jcerdi@@itam.mx. Agradezco la valiosa y enriquecedora discusión de las ideas aquí presentadas al profesor Germán Sucar; así como a los asistentes al seminario de doctorado organizado por la Universidad de la Magna Grecia en Catanzaro y a los asistentes al ciclo de conferencias de la maestría en Filosofía del Derecho de la Facultad de Derecho de la Universidad de Buenos Aires.
}

Este artículo fue recibido el 6 de septiembre de 2013, siendo aprobada su publicación el 14 de mayo de 2014. 
Cerdio - Razonar en lo jurídico y en lo moral. Dos razones, diferentes racionalidades

(deciding what to do in a particular case). Such requirements reveal that some notions of rationality are applicable to legal reasoning but not to its moral counterpart.

Keywords: legal reasoning - theoretical reasoning - practical reasoning - moral practical reasoning.

\section{Introducción}

Se sostiene la tesis de que el análisis del razonamiento jurídico requiere la distinción entre dos tipos de racionalidad: la racionalidad teórica y la racionalidad práctica. ${ }^{1}$ Sostendré incluso que el razonamiento jurídico práctico bajo ciertas condiciones ${ }^{2}$ presupone el uso de la razón teórica (concerniente al conocimiento de normas y al conocimiento de los hechos, a los que llamaré de aquí en más conocimiento de normas $\mathrm{y}$ de hechos, respectivamente).

Estas características del razonamiento jurídico son relevantes para diferenciarlas del razonamiento moral. Este último, en efecto, constituye un ejercicio exclusivo de la razón práctica. Una de las consecuencias de aceptar esta característica del discurso moral es que la evaluación de los razonamientos prácticos en contextos morales (razonamiento práctico moral) adopta la forma de un particularismo ${ }^{3}$ cuando se trata de la reflexión que hacen los agentes acerca del curso de acción a tomar. Es decir, el examen y evaluación de la conducta que desplegará el agente -O que ha desplegado, si se trata de una retrospectiva- es irremediablemente relativo al contexto de la situación, circunscrito a un momento de decisión individual e irrepetible, no generalizable, invariablemente revisable y limitado en su racionalidad.

Para argumentar la tesis anterior primero se distingue entre razón práctica y razón teórica. En segundo lugar se define razonamiento práctico bajo un doble nivel de análisis -la reflexión y la inferencia práctica- y se presenta un esquema de razonamiento para el dominio moral. En tercer lugar se argumenta a favor de mantener la separación entre razón práctica y razón teórica como un supuesto deseable del esquema del razonamiento práctico moral (tesis de la separación). Después de ello se analiza en qué sentido se puede predicar racionalidad del razonamiento práctico moral. Finalmente se emplean las consideraciones anteriores para distinguir entre el razonamiento jurídico y el razonamiento práctico moral.

\footnotetext{
${ }^{1}$ WiLLIAMs (1972), p. 55.

${ }^{2}$ Las condiciones normativas que le impone la constitución al legislador para la creación de normas o las que impone la Constitución y la ley a un juez de motivar y justificar sus sentencias.

3 La expresión particularismo está inspirada aquí en una interpretación libre de las tesis del denominado particularismo moral, tal y como lo ha presentado y defendido Dancy. DANCY (2004); LITTLE (2001).
} 


\section{Dos racionalidades}

Se pueden distinguir dos tipos de preguntas filosóficas de naturaleza excluyente: (1) ¿Qué hay en el mundo? y (2) ¿Qué se debe elegir en el mundo? La primera pregunta ocurre en el dominio de lo que se ha llamado razón teórica. Una de las maneras de traducir la primera cuestión es qué creencias acerca del mundo son verdaderas. La segunda pregunta se equipara a la cuestión de determinar qué cursos de acción tomar en diferentes contextos y cae en el dominio de la razón práctica. Las dos preguntas son distintas en tanto que las respuestas satisfactorias a la primera pregunta no implican responder a la segunda pregunta -saber qué es el caso no implica saber qué hacer en el caso o con relación al caso-.${ }^{4}$ Por ejemplo, saber que es verdad que "Romeo está envenenado" no implica que el médico que recibe al mismo tiempo a Romeo y a Julieta que también está envenenada y solo puede materialmente atender a uno de ellos, sepa qué hacer. $\mathrm{O}$, en otros contextos menos dramáticos, del hecho de saber que en una tienda de ropa hay 45 modelos de prendas de vestir, todos de la talla que nos acomoda, no nos dice nada acerca de cuál modelo elegir. Que un agente sepa que ciertos enunciados acerca del mundo son verdaderos no implica que sepa (ni que de hecho realice) el curso de acción correcto. Tampoco es válida la afirmación inversa: elegir el curso de acción correcto no implica conocer la verdad de ciertos enunciados acerca del mundo. Así por ejemplo, una madre sabe que debe proveer de remedio a su hijo enfermo. Este saber no implica que sepa qué enfermedad tiene, su cura, el pronóstico ni lugar en donde pueda ser atendido. Que la madre haya elegido el curso de acción adecuado no implica que sepa cómo llevar a cabo dicho curso de acción. En suma, un tipo de razón no implica a la otra.

De otro lado, la separación entre las dos racionalidades también se aprecia en que la elección de un curso de acción se puede juzgar por una variedad de criterios, ninguno de los cuales es aplicable para juzgar el conocimiento que tenemos del mundo. Entre los criterios para juzgar la elección entre alternativas se encuentra: el beneficio que reporta (elegir la profesión mejor remunerada), la

\footnotetext{
${ }^{4}$ Conviene no confundir la separación entre los criterios para responder a las preguntas ¿Qué hay en el mundo? y ¿Qué hacer en el mundo? con la separación que hay entre saber y saber hacer. Esta última separación va dirigida a distinguir entre la verdad de ciertos enunciados y las habilidades que se despliegan usando el contenido de los enunciados. Es diferente saber la lista de ingredientes y procedimientos descritos en una receta y saber hacer - ejecutar- la receta, saber las reglas del fútbol y saber jugar fútbol, etcétera. Entre las dos formas de saber existe una relación de precedencia: la habilidad que despliega quien sabe hacer implica que ha tenido conocimiento, que sabe técnicamente lo que se requiere. Un jugador de fútbol sabe las reglas y la definición y propósito del juego cuando juega al fútbol. En cambio, la distinción entre razón teórica y razón práctica no apunta a la distinción entre conocimiento proposicional y conocimiento práctico, sino a la distinción entre los criterios que tenemos para identificar las creencias verdaderas y los criterios que tenemos para evaluar cursos de acción elegidos por los agentes. Un tipo de racionalidad no antecede a la otra, ni la implica. STANLEY y WiLLIAMSON (2001), pp. 433-434.
} 
satisfacción de una meta que se adopta (llegar a tiempo a trabajar), la coherencia con ciertas apetencias (siempre que se escoja pescado se elige un vino blanco) o el acuerdo previo con otros (se acuerda con el socio comercial elegir el insumo más barato). Pero ninguno de estos criterios es conceptualmente aplicable a la identificación de creencias verdaderas. Sería un error de categoría $a^{5}$ decir que "es verdad que X es el caso" porque reporta más beneficio, satisface una meta, es coherente con mis apetencias o, finalmente, porque lo he acordado con otros. ${ }^{6} \mathrm{La}$ afirmación contraria también es válida: los criterios para determinar la verdad de una creencia - o poseer una creencia verdadera acerca del mundo- no implican por sí mismos un beneficio, ni la satisfacción de una meta ni la coherencia con ciertas apetencias ni el acuerdo previo con otros. En conclusión, la razón teórica se distingue de la razón práctica, también, en que los criterios que son aplicables a un dominio de la razón no son aplicables al otro.

Por cierto que la separación entre las dos racionalidades no implica que no existan conexiones entre ellas en contextos específicos. Así, en ciertos contextos de elección los agentes deben justificar la adopción de un curso de acción bajo criterios que incluyen mostrar que actuó bajo creencias verdaderas: la justificación del médico en el uso de un antibiótico incluirá, cuando menos, la creencia de que es verdad que el paciente padecía de una infección -y que el antibiótico es adecuado para la infección-, la justificación de una madre que le reprocha a su vecino la agresión que le propinó a su hijo incluye, cuando menos, creer que el vecino fue el autor de la agresión, la decisión de un piloto de aterrizar de emergencia por falta de combustible incluye, cuando menos, creer que es verdad que el avión no tenía combustible suficiente para llegar al lugar de destino. En todos estos ejemplos la conexión entre las dos racionalidades se muestra porque la racionalidad práctica presupone cuando menos la existencia de ciertos objetos, hechos o situaciones y, por el otro, de relaciones de medio a fin. Esto muestra, también, que el conocimiento teórico no está exento de tener finalidades prácticas - por ejemplo, investigar el mecanismo de una enzima para generar una cura contra el cáncer-.

\section{Razonamiento práctico}

El razonamiento práctico puede ocurrir en un sinnúmero de contextos prácticos, cotidianos (elegir el atuendo adecuado para una fiesta), técnicos (decidir acerca del mejor tratamiento para un paciente) o morales (escoger entre salvar la vida del inocente o cometer un homicidio). En todos estos contextos puede estar presente tanto un discurso como un proceso psicológico.

\footnotetext{
${ }^{5}$ RYLE (2002), p. 16.

${ }^{6} \mathrm{La}$ aplicación de los criterios para evaluar las elecciones puede apoyarse en la verdad de ciertos enunciados acerca del mundo: que es verdad que una actividad es lucrativa, que algo es un medio para llegar a tiempo, que la comida servida es pescado, que el insumo a comprar es el de menor precio.
} 
Interesa la racionalidad práctica para analizar el razonamiento cuando se eligen cursos de acción en un sentido discursivo. Es decir, interesa analizar el razonamiento práctico en tanto un discurso que elaboran los agentes en contextos de decisión práctica. No interesan, en cambio, los procesos psicológicos que conducen a una decisión o a la percepción del agente de que su conducta está justificada, estos son fenómenos de la psicología.

En tanto discurso de justificación, el razonamiento práctico tiene dos momentos o niveles de análisis: la reflexión acerca de los bienes, valores o fines que han de constituir la premisa mayor de su razonamiento, y acerca de la situación en que ha de decidir, por un lado, y la inferencia práctica, por la otra. La reflexión práctica surge cuando el agente se pregunta por los cursos de acción disponibles, los objetivos prácticos que persigue (o que puede perseguir) con cada uno de ellos, los fines que justifican ${ }^{7}-\mathrm{O}$ no- los objetivos trazados, los medios que son adecuados para satisfacerlos, así como de su corrección normativa. En esta etapa considerará asimismo si los distintos objetivos que persigue son o no incompatibles -ya sea para descartar alguno de ellos, jerarquizarlos o reformularlos-, el horizonte temporal que enmarca la decisión y también el grado de compromiso - interés ${ }^{8}$ que tiene con la situación.

Una vez concluida la reflexión práctica, el agente puede esquematizar su resultado como un conjunto de premisas que al ser aceptadas permiten concluir un curso de acción para el caso. Denominaremos esquema de razonamiento práctico a dicha esquematización (ERP). El ERP se puede ilustrar como sigue:"

\footnotetext{
${ }^{7}$ Los fines han de entenderse en sentido normativo: sirven para evaluar los objetivos prácticos trazados. Por ejemplo, un agente tiene el objetivo de acumular dinero. La evaluación puede recaer en el objetivo a partir de una finalidad no admisible, o un sentido normativo: conseguir poder, lograr control sobre otros, desposeer de sus bienes a terceros. Por cierto que el objetivo práctico trazado puede ser también moralmente incorrecto sin el empleo de finalidades: el objetivo práctico de dañar a otro por placer.

${ }^{8} \mathrm{El}$ horizonte temporal en el que se proyecta el problema práctico incide en la reflexión no es lo mismo proyectar el problema de qué hacer con la moneda que se tiene aquí y ahora respecto de un indigente o proyectar esta pregunta sobre el día de mañana o los siguientes 20 años de vida del desvalido. El mayor o menor compromiso que el agente tenga con la situación incide de igual forma en la reflexión práctica. Así, por ejemplo, se puede aceptar que el dinero es mejor empleado para aliviar la pérdida de quien ha sufrido una desgracia -los damnificados de un país lejano y desconocido- que en la compra de una mascota. Pero si se trata de emplear el dinero para comprar una mascota de reemplazo para aliviar el mal rato que pasa el hermano o la hermana, la situación que padece el ser querido parece incidir en nuestra decisión moral; el sufrir del ser querido parece más próximo que el sufrimiento de una población desconocida. Esto obedece a la posición personal y al interés que el agente guarda con el problema. En definitiva no es lo mismo decidir acerca de lo que está lejano en el tiempo -sea pasado o futuro- y lejos de nuestro círculo cercano de intereses y afectos que de aquello que es inmediato y cercano a nosotros. GuibOuRG (1999), p. 34.

9 También se debe distinguir el razonamiento práctico de la explicación teleológica de la conducta. Esta última es un metadiscurso expost que da cuenta de los motivos o causas que determinaron la conducta del agente. En cambio, el razonamiento práctico tiene una finalidad justificativa. Sea que
} 
Cerdio - Razonar en lo jurídico y en lo moral. Dos razones, diferentes racionalidades

1. Enunciado que establece que el agente $\mathrm{A}$ desea alcanzar el fin $\mathrm{F}$ (enunciado de fines).

2. Enunciado que expresa que $M$ es un medio para alcanzar $F$ (enunciado de medios).

3. Enunciado que expresa que el agente debe realizar un acto (o abstención) que puede ser descrito como M (conclusión práctica).

Las dos fases del razonamiento práctico están relacionadas a modo de un proceso y un producto, porque las premisas de la inferencia práctica son el producto de la reflexión práctica (balance de razones). La etapa de reflexión podría ser expresada a modo de un esquema argumental con premisas que se ofrecen para justificar una conclusión práctica, de modo que la reflexión es una tarea previa a la fase de inferencia. ${ }^{10}$

Otra diferencia entre las dos fases del razonamiento práctico está dada por los criterios que sirven para evaluarlas.

Por lo que hace a la etapa de reflexión puede haber ciertos criterios de racionalidad que sirvan para evaluarla, como veremos más adelante (sección 4).

Por lo que hace a la inferencia práctica, habrá tanto un criterio formal como uno material de evaluación. Desde un punto de vista formal, la corrección de la inferencia práctica está dada por la noción de consecuencia lógica: ${ }^{11}$ si la conclusión se sigue en forma lógica de las premisas, entonces la inferencia será formalmente correcta. Materialmente la corrección se predica de cada una de las premisas de la inferencia práctica, es decir, de si los fines propuestos están justificados y si los medios elegidos son adecuados -y justificados-. Como la justificación es una relación entre un dominio de razones y un conjunto de fines, la justificación de la premisa mayor es relativa al dominio de razones. ${ }^{12} \mathrm{~A}$ este respecto, un fin está justificado de uno de los dos modos, o bien porque el agente,

el razonamiento práctico ocurra antes de que realice un curso de acción, cuando el agente delibera lo que debe elegir o que ocurra cuando el agente ha realizado el curso de acción -en retrospectiva-. En ambos casos el razonamiento no va encaminado a explicar la conducta a realizar -o realizada, sino a su justificación. Adicionalmente, el razonamiento práctico adopta un punto de vista en primera persona del agente, comprometido, interno. Por oposición, la explicación teleológica adopta un punto de vista que suele ser en tercera persona, descomprometido, externo a la decisión. Sin perjuicio de que es posible adoptar un punto de vista externo, descomprometido, hipotético acerca de lo que se haría si se estuviese en la situación, en primera persona. VON WRIGHT (1963), p. 11; VON WRIGHT (1979), p. 121.

${ }^{10}$ Sin que esto implique que en todas las ocasiones de una inferencia práctica el agente volverá a revisar la etapa de reflexión. Puede ocurrir que el agente esté seguro del balance de razones hecho en el pasado y continúe con él hasta en tanto no encuentre una situación que le sugiera una revisión. BRATMAN (1987), p. 218.

${ }_{11}$ Para la idea de criterio formal basta con una noción abstracta de consecuencia lógica que permita manipular premisas normativas y fácticas. ALCHOURRÓN y MARTINO (1990), pp. 46-67.

12 CARACCIOLO (2009), p. 43. 
al menos, suscribe que el fin está justificado en relación con sus deseos, preferencias e intereses (dominio interno de razones) o bien porque suscribe que el fin está justificado en relación con un hecho externo, en la forma de lo que otros recomiendan o prescriben, o de un beneficio colectivo o de un acuerdo convencional (dominio externo de razones). ${ }^{13}$

De este modo, la premisa mayor de la inferencia práctica se entiende justificada cuando el fin que expresa está justificado.

Por su parte, la premisa menor de la inferencia práctica expresa una relación anankástica, entre una situación, hecho o conducta y el fin suscrito como justificado por el agente. La justificación de esta premisa estará dada en función de la verdad de la proposición que describe la relación medio a fin y de la corrección de los medios elegidos.

Nótese que tanto en el caso del conjunto de creencias o razones que llevó al agente a seleccionar la premisa mayor o la premisa menor, a la postre puede resultar insuficiente o erróneo y, en última instancia, no justificado. Con ello, que la conclusión se desprenda formalmente de las premisas y que estas estén justificadas a la luz de las creencias o razones del agente al momento de efectuar el balance de razones no implica que dicho balance pueda, a la postre, en un nuevo análisis, ser revisado y determinarse como incompleto, insuficiente, erróneo, en suma, no justificado. Es inevitable que todo balance de razones esté sujeto a revisión y, por tanto, la corrección material de la inferencia práctica. ${ }^{14}$ Dicho de otro modo, no existe un balance de razones invariablemente correcto, pétreo, hacia el futuro -que sea transversal mediante cualquier contexto- ${ }^{15}$ Como la idea

\footnotetext{
${ }^{13}$ La idea de que la justificación es una relación entre un dominio de razones y un conjunto de conductas o situaciones es compatible con adoptar un internalismo o un externalismo moral, porque basta con distinguir entre la tesis que dice que la justificación requiere mostrar dos extremos de una relación -razones y conductas o situaciones- de las tesis que postula el origen de las razones -internalismo o externalismo-. BAYÓN (1991), p. 76; WILLIAMS (1981), pp. 101-13.

${ }^{14} \mathrm{La}$ idea de que un balance de razones está invariablemente sujeto a revisión es consecuencia de aceptar que todo razonamiento práctico parte de una etapa de reflexión en el que el agente se cuestiona acerca de qué curso de acción tomar habida cuenta de los objetivos, fines, medios y razones. La idea de una razón excluyente, que proscribe la reapertura de un balance de razones futuro, es compatible con la presentación aquí hecha de una etapa de reflexión: en la ocasión siguiente a la adopción de la razón excluyente el agente deberá reflexionar y decidir no abrir el balance de razones. Se puede decir que aceptar estas razones - excluyentes- implica que en cada caso de reflexión se hará la revisión de la situación para admitir que se adoptará una actitud de no apertura del balance. Y que esto es, también, una decisión. De otro modo se seguiría que del hecho de que una razón es excluyente esto implica que los agentes actuarán de la misma manera que ella, como una consecuencia lógica: lo que se debe hacer implica lo que se hace. RAZ (1990), p. 39.

15 Es compatible con la idea de revisar el balance de razones, que en un balance de razones particular, el agente concluye que debe mantener el resultado de su balance en la etapa de reflexión a lo largo del tiempo, a modo de un compromiso. Por ejemplo, el consultor de tecnología que descubre que ha procedido en el pasado bajo el influjo del prejuicio en contra de la tecnología hecha en la India y resuelve, en todos los casos futuros, en que tenga que decidir qué tecnología
} 
de balance de razones incluye la reflexión acerca de los objetivos prácticos a la luz de ciertos fines normativos, los medios y su idoneidad respecto de los objetivos y su corrección, que todo balance de razones sea sujeto a revisión posterior implica que no hay medios ni objetivos inmunes a revisión, invariablemente correctos, para todos los contextos, tan solo porque no es imposible su enmienda o descarte producto de una nueva revisión.

\section{Razonamiento práctico moral}

El razonamiento práctico en el contexto moral también posee dos etapas de su análisis: reflexión e inferencia práctica. En esta última se presenta una especificación propia del dominio moral según se admita un deber moral genérico de hacer lo que es moralmente correcto. Cuando así se admite, la inferencia práctica adopta una forma discursiva de justificación práctica para cualquiera que acepte la prescripción de que "se debe hacer lo moralmente correcto y abstenerse de hacer lo moralmente no correcto". Esta aceptación introduce una variante respecto del ERP y que produce una nueva forma de esquematización de la inferencia práctica que denominaremos razonamiento práctico moral (RPM). El RPM puede ser representado mediante el siguiente esquema:

1) El agente A desea hacer lo que es moralmente correcto (enunciado de fines).

2) M es una conducta (situación) genérica que es moralmente correcta (enunciado de medios).

3) Si $\mathrm{m}$ puede ser descrita como es una instancia de $\mathrm{M}$, entonces $\mathrm{A}$ debe hacer $\mathrm{m}$ (conclusión práctica).

En común con el ERP, el RPM tiene que ambos son esquemas de la inferencia práctica: la conclusión es consecuencia de las premisas, en una relación de inferencia, que admite ser evaluada por su corrección formal. De otro lado, también el RPM es el producto de la reflexión del agente acerca de los fines y medios que juzga relevantes, de las razones pertinentes para justificar la adopción de unos y otros - corrección de fines, idoneidad y corrección de medios- y, en definitiva, de la elección del curso de acción. Como cada una de estas consideraciones puede ser revisada en una ocasión futura, se sigue que todo balance de razones generado en la etapa de reflexión está sujeto a revisión y eventual modificación o rechazo. ${ }^{16}$ De modo que también se puede juzgar, ex post,

recomendar, operar bajo la presunción de que los productos de la India son confiables. También es compatible la idea de la revisión del balance de razones con concluir que se debe realizar una actividad a lo largo del tiempo: quien resuelve que de ahora en más hará 20 minutos de ejercicio por las mañanas por el resto de sus días. En ambos escenarios, frente a un caso futuro, el agente incluirá su determinación pasada en el balance de razones presentes.

${ }^{16}$ Aquí conviene trazar una semejanza entre la idea de que todo balance de razones generado en la etapa de reflexión es sujeto a revisión -y eventual modificación, rechazo o confirmación- y una 
la corrección material de las premisas de un determinado RPM en el mismo sentido en el que se puede juzgar corrección material del ERP.

En cambio, una diferencia entre el ERP y el RPM es que el fin práctico que se persigue es realizar conductas moralmente correctas $-\mathrm{y}$ abstenerse de las incorrectas-. En consecuencia, cualquier conducta que sea un medio para realizar ese fin debe -o debe no- ser realizada. La inferencia práctica estará orientada hacia alcanzar este fin general. En este aspecto, el razonamiento práctico moral está específicamente orientado hacia la consecución de un fin determinado -genérico-. Al mismo tiempo, como la determinación acerca qué objetivos prácticos y qué cursos de acción conllevan alcanzar el fin se realiza en cada caso concreto y es el producto de la reflexión, el razonamiento práctico es abierto: el agente debe reflexionar sobre la relación entre los cursos de acción que se le presentan en la situación concreta con miras a hacer lo moralmente debido, en cada ocasión.

De este modo es que la etapa de reflexión está orientada hacia el análisis de la situación concreta que enfrenta el agente, hacia los distintos cursos de acción posibles entre los que debe optar con miras siempre a alcanzar el fin de hacer lo moralmente debido y evitar lo moralmente indebido. También se puede reflexionar sobre si el objetivo práctico que se persigue es compatible con el deber moral genérico asumido. Por ejemplo, se puede preguntar si el objetivo de controlar una red de funcionarios para tenerlos a disposición de una empresa es un objetivo compatible con la finalidad genérica de "hacer lo moralmente correcto y abstenerse de hacer lo moralmente no correcto". O bien, la reflexión se puede dirigir hacia la relación entre un curso de acción y un objetivo que ya se juzgó compatible con el deber genérico asumido. Por ejemplo, se juzgó compatible con la finalidad de hacer lo moralmente debido ayudar al hermano que pasa por una mala situación financiera, pero se reflexiona sobre el medio más adecuado: entregarle personalmente dinero, buscar un benefactor anónimo, allegarle de información acerca de posibles empleos, asistirle con alimento y vestido. Todo esto en el contexto de un hermano menor, pródigo, muy orgulloso y que guarda escaso contacto con nosotros. $\mathrm{O}$, finalmente, puede que la reflexión se centre

tesis de alguna versión del particularismo moral. A saber, la tesis de que no se pueden identificar propiedades moralmente relevantes, cuya relevancia sea invariable para todos los contextos prácticos. Es decir, que la identificación de una propiedad moral, con relevancia para perseguir el fin genérico de hacer lo moralmente debido -y evitar lo moralmente indebido-, no se puede dar ex ante a la reflexión frente al caso particular ni se puede clausurar el juicio sobre su relevancia ex post, una vez terminada la reflexión (el balance de razones) para cualquier contexto futuro. Lo que se podría hacer, en todo caso, en una nueva situación, es confirmar la relevancia de la propiedad que se juzgó en un caso pasado -p.ej. mentir es moralmente relevante-, lo que supone aceptar que la relevancia no fue aceptada ex ante, sino a partir de la reflexión de la nueva situación. La comparación entre el carácter revisable del balance de razones y esta tesis atribuible a alguna versión del particularismo se inspira en la discusión que presenta Shafer-Landau acerca del papel que cumplen las reglas morales en la identificación de propiedades moralmente relevantes - p.ej. la regla que dice que "Mentir es moralmente indebido" identifica la propiedad mentir -o la mentiracomo moralmente relevante. SHAFER-LANDAU (1997). 
Cerdio - Razonar en lo jurídico y en lo moral. Dos razones, diferentes racionalidades

tanto en la compatibilidad del objetivo práctico con el deber de hacer lo moralmente correcto como en la corrección de los medios.

Una vez terminada la etapa de reflexión, la inferencia práctica moral incluirá un enunciado de medios que refleje la relación entre la situación concreta reflexionada y el cumplimiento del deber genérico: "Mentir es moralmente indebido", "Ayudar al desvalido es moralmente debido", "Lesionar a otro por placer es moralmente indebido".

El razonamiento práctico moral, con sus dos etapas - de reflexión y de inferencia práctica moral no prejuzga sobre la ontología ni el tipo de epistemología del dominio moral y es compatible con un sinnúmero de posturas no solo metaéticas, sino incluso de éticas de primer orden. Lo que supone, en cambio, en primer término, es la aceptación de que los razonamientos prácticos en contextos morales están compuestos de enunciados de fines, enunciados de medios y una conclusión normativa. Pero sobre todo, adopta la tesis de que el razonamiento práctico moral así concebido se diferencia de la razón teórica-qué creencias son verdaderas- en tanto persigue establecer qué curso de acción elegir en un caso individual -el curso de acción moralmente correcto-.

Es decir, se sostiene la tesis de que el razonamiento práctico moral pertenece al dominio de la razón práctica y no al de la razón teórica y, lo que es más significativo, para nuestros propósitos, no presupone -a diferencia del razonamiento práctico jurídico- una previa tarea cognoscitiva vinculada a los estándares normativos -regla, norma, principio o razón- y a los hechos relevantes del caso.

En primer término, no hay una exigencia de conocer, identificar un conjunto de estándares normativos, ni existen estándares que se refieran a otros estándares para delimitar ámbitos de aplicación -por razón del lugar, las personas o las conductas- o para establecer la aplicabilidad de los estándares a ciertos casos, p.ej. vigencia del estándar o vinculación de los casos precedentes. ${ }^{17}$

Tampoco existe una exigencia respecto del conocimiento de los hechos, por ejemplo, tener algún grado de conocimiento de lo ocurrido -conocer por oposición a presumir-, o las fuentes de conocimiento que se pueden emplear para conocer la situación -empírica, autoridad, cálculo, inducción, intuición-, o exigencias acerca del modo de allegarse de la información -mediante terceros o por cuenta propia-, ni, finalmente, existe una limitación acerca del tipo de hechos a considerar en la etapa de reflexión, p.ej. únicamente se pueden considerar hechos o acciones pasadas, pero no futuras, situaciones reales, pero no hipotéticas, acciones propias, pero no de terceros.

Denominaremos tesis de la separación a la idea que sostiene que el razonamiento práctico moral pertenece al dominio de la razón práctica y no al de la

${ }^{17}$ NAVArro, ONORESU, RodríGuEZ y SuCAR (2001), pp. 133-152. 
razón teórica y que, en consecuencia, no presupone una previa tarea cognoscitiva. Por "tarea previa cognoscitiva" se entiende que sea una condición necesaria que el agente averigüe la verdad de sus creencias acerca de los estándares morales y de los hechos que se le presentan en la situación práctica como una exigencia conceptual anterior a la reflexión y a la inferencia práctica. Que esta tarea cognoscitiva no sea presupuesta para el razonamiento práctico moral también quiere decir que la actividad de decidir qué creencias acerca de dichos estándares y hechos son verdaderas tampoco es una exigencia fáctica para realizar la reflexión y la inferencia práctica. Es decir, que en el razonamiento práctico moral no se presupone ni conceptual ni fácticamente una tarea cognoscitiva previa acerca de los estándares normativos y de los hechos relevantes del caso. ${ }^{18}$

La negación de la tesis de la separación es que la elección moral es una tarea propia del dominio de la razón teórica. Saber las creencias verdaderas acerca de lo que es correcto es una condición necesaria -conceptual o fácticamente previa- para el razonamiento práctico, es decir, para elegir el curso de acción correcto. Denominaremos a la negación de la tesis de la separación, la tesis de la conexión. En su versión fuerte, la tesis de la conexión dice que es imposible, conceptualmente, realizar la tarea de elegir el curso de acción correcto sin conocer la verdad moral acerca del caso. En su versión débil, la tesis dice que es imposible, fácticamente, que un agente elija el curso de acción correcto sin conocer la verdad moral para el caso.

Podemos decir que la tesis admite varias formas de especificación según el contenido del conocimiento que se juzga indispensable para la elección del curso de acción correcto. Este contenido puede ser una virtud, una habilidad, una intuición, un cálculo y, en especial, un estándar normativo, regla, principio, valor. Interesa analizar la tesis de la conexión -fuerte o débil- bajo la forma de especificación que sostiene que el agente requiere conocer ciertos estándares normativos para realizar una elección correcta en el caso. Esta forma de especificar la tesis de la conexión rezaría como sigue: si un agente posee una creencia verdadera acerca de la existencia y contenido de los estándares normativos que regulan el caso entonces también sabe qué curso de acción es el moralmente correcto (tesis de la conexión por estándar). Es decir, conocer el catálogo de estándares normativos, morales, implica que se puede inferir qué hacer en los contextos prácticos relevantes. ${ }^{19}$

\footnotetext{
18 Existen, sin embargo, importantes relaciones entre el razonamiento práctico y la verdad de ciertas creencias cuando se realiza la reflexión. En la medida en que el razonamiento práctico moral es también un razonamiento práctico, en esa medida mantiene las relaciones y funciones de averiguar la verdad de ciertas creencias. Por ejemplo, se puede reprochar a quién procede a tomar una decisión moral ignorando hechos que puede conocer y que decide desechar: las consecuencias de una acción, el estado emocional de un tercero, las necesidades básicas de otros, la manifestación de intenciones y deseos de los agentes, etcétera. Véase arriba nota 6.

19 MOORE (2010); GEORGE (2002).
} 
La tesis de la conexión por estándar desconoce la distinción entre razón teórica y razón práctica para el dominio moral porque saber qué creencias son verdaderas -conocer los estándares normativos morales- es condición para escoger el curso de acción correcto. La idea general detrás de la tesis de la conexión por estándar es que conocer los estándares normativos es condición de la elección del curso de acción moralmente debido. Saber qué hacer en el caso -elegir el curso de acción moralmente correcto- implica saber qué estándares regulan el caso. La tesis es compatible con afirmar que un agente que conoce el estándar normativo no elige de becho el curso de acción correcto - p.ej. el estándar indicaba que mentir es moralmente indebido y, frente a la ocasión para mentir, el agente miente. Esto es así porque la tesis no indica que lo que debe ser elegido por el agente siempre sea de hecho lo que el agente hace. En cambio, sostiene que si un agente ha elegido el curso de acción moralmente correcto - p.ej. abstenerse de mentir- es porque ha conocido la existencia y contenido de un estándar moral -p.ej. "mentir es moralmente indebido".

La primera objeción que se puede hacer a la tesis de la conexión por estándar es que el conocimiento de un estándar normativo no justifica la elección de un curso de acción. Los estándares normativos indican lo que se debe hacer, norman situaciones o califican como moralmente debida una conducta $-\mathrm{y}$ en este sentido modalizan deónticamente las conductas. La calificación de una conducta como moralmente debida no es igual a la actividad de justificar la elección de esa conducta. La justificación del curso de acción elegido es provista por el balance de razones, las razones que se ofrecen para realizar la acción, las que acepta el agente. Por cierto que la etapa de reflexión puede incluir el estándar moral, en tanto se reflexiona si hay razones para seguirlo: el modo en que regula la conducta, la calificación que el estándar hace de la conducta o la justificación subyacente al estándar. Se trata de que el agente incluya razones acerca del estándar, internamente en el balance de razones. El razonamiento práctico en tanto discurso de justificación exhibe las razones que el agente aceptó para elegir el curso de acción. La justificación es una actividad discursiva y no una propiedad -lógica, natural o lingüística- de los estándares normativos. Esto es, los estándares no justifican, justificar es una actividad discursiva que hace el agente cuando razona y que puede incluir, también, algún estándar como objeto de su razonamiento. Cuando un estándar es considerado en la reflexión práctica, la justificación recaerá sobre la procedencia del estándar, su justificación subyacente, el alcance de su contenido u otras consideraciones más. Pero en ningún caso justificar una elección entre cursos de acción es equivalente a conocer un estándar ni la existencia del estándar implica la justificación de la conducta requerida por el estándar ni, finalmente, la justificación de la conducta elegida por el agente.

La segunda objeción es que la tesis de la conexión debe mostrar que del contenido del estándar general se sigue conocer $-\mathrm{O}$ reconocer- su aplicación en el caso concreto, saber aplicar el estándar moral. Pero este no parece ser el caso: todo estándar general requiere de una operación de aplicación. Esta es una 
característica $-\mathrm{y}$ un problema filosófico- de todo estándar general: ${ }^{20}$ que requiere de una habilidad para su aplicación en un caso concreto. En efecto, la operación de aplicar un estándar normativo a un caso individual supone otras tareas distintas que no son equivalentes a la mera tarea cognoscitiva de identificación del estándar y su contenido: (1) el agente debe resolver si más de un estándar es aplicable al caso y ordenarlos entre sí, en caso de que más de uno sea aplicable, (2) reconocer que el caso individual que tiene frente a sí está regulado por los estándares identificados ${ }^{21}$ y, por último, (3) aceptar que el estándar es concluyente, sin excepciones, ${ }^{22}$ frente a las instancias que regula (habida cuenta de su peso y relevancia específica). De modo que la tesis de la conexión implica que los estándares normativos morales a diferencia de otro tipo de estándares normativos -jurídicos, religiosos, médicos, ingenieriles- no requieren de ninguna operación por parte del agente para su aplicación.

La tesis que se sostiene aquí, en cambio, es la tesis de la separación; que implica que los criterios parar discernir entre creencias verdaderas y falsas no son aplicables al contexto de la elección de cursos de acción. Hay que hacer énfasis en que la tesis de la separación conlleva que en la primera etapa de reflexión no se tiene una guía ex ante del curso de acción a elegir ni una predeterminación independiente de la actividad de reflexión y del balance de razones que efectúa el agente. $^{23}$

En la primera etapa de reflexión, en efecto, el agente se supone abierto a considerar todos los argumentos que estime pertinentes para identificar las propiedades relevantes en el contexto, para considerar la calificación que le merece cada propiedad, el modo en que intervienen cada una de las propiedades y su calificación, de forma que le permita obtener un resultado, en lo ideal, concluyente acerca de qué fin perseguir y por qué medios que sean fácticamente adecuados y normativamente correctos. En la primera etapa de reflexión la relevancia de las

\footnotetext{
20 O puesto de otro modo: se trata del problema de saber si una conducta individual constituye un cumplimiento del estándar o una conducta contraria a lo que el estándar exige. El saber proposicional no puede suministrar una respuesta a este problema. Por cierto que se puede decir que los practicantes muestran, con su conducta, el estándar y lo que requiere. Pero como hemos dicho, el saber hacer -incluso el relativo a la aplicación de estándares- no ha de ser confundido con la racionalidad práctica. GREENAWALT (2001).

${ }^{21}$ CARACCIOLO (2009), pp. 145 y ss.

${ }^{22}$ Como se ha argumentado, una excepción es relativa a un tipo de decisión y a un contexto. En el contexto de la decisión moral general -hacer lo moralmente correcto- y habida cuenta de que el agente debe abrir una reflexión para considerar qué hacer en el caso, la operación de distinguir una situación según un estándar de una situación que cuenta como una excepción no está implicada en el conocimiento que el agente tiene del contenido del estándar general. Es decir, el conocimiento proposicional de un estándar no implica la operación de identificar una excepción y, por tanto, un caso de aplicación no excepcional. DUARTE D'ALMEIDA (2013).

23 Una cuestión interesante -que no discutiré aquí- es poner a prueba la tesis de la separación frente a la proposición que dice que existen algunos balances de razones morales que permiten conocer el curso de acción correcto en un caso. Es decir, poner a prueba la tesis de la separación frente a una concepción epistémica del balance de razones.
} 
Cerdio - Razonar en lo jurídico y en lo moral. Dos razones, diferentes racionalidades

propiedades no está dada por la existencia ex ante de estándares normativos, sino que dichas propiedades, como su calificación, son el resultado de un balance de razones sobre los fines y los medios para satisfacerlos, en función de cierta situación -aunque el balance también puede versar sobre la existencia, contenido o propósitos de un estándar normativo- ${ }^{24}$

\section{Racionalidades del razonamiento práctico moral}

Que el razonamiento práctico moral sea abierto y relativo al contexto en su etapa de reflexión no implica que la discusión y evaluación del balance de razones sea irracional o carente de criterio. Por el contrario, es posible defender criterios de corrección racionales para evaluar el discurso de justificación del balance de razones. La racionalidad de una discusión acerca de la etapa de reflexión en el razonamiento práctico y los resultados a los que se arriban -elección de fines y medios- requiere aclarar el sentido en que una discusión -o una revisión- de tal naturaleza revisionista puede ser racional.

En un primer sentido, la etapa de reflexión del razonamiento práctico es racional porque el agente se conduce tratando de dilucidar qué le exige la moral en la situación práctica. En este modo de proceder, la palabra racional podría querer decir, en primer lugar, que la pregunta por los cursos de acción correctos tiene sentido de ser formulada y, en consecuencia, también tiene sentido buscar una respuesta con relación a ella. La idea de una reflexión racional es analítica a la concepción del razonamiento práctico aquí presentada. Cada vez que un agente realiza la etapa de reflexión en un razonamiento práctico está siendo racional.

Una reflexión moral podría ser racional, en segundo lugar, cuando el agente conduce el discurso de reflexión sin inconsistencia: ${ }^{25}$ identifica los fines que regulan la justificación de sus objetivos prácticos y acomoda las incompatibilidades ${ }_{-} \mathrm{O}$ advierte los dilemas irresolubles- entre los fines, identifica y determina la compatibilidad y factibilidad de los medios. En este segundo sentido la etapa de reflexión es racional en tanto es conducida bajo el ideal de la consistencia.

\footnotetext{
${ }^{24}$ Las premisas de la inferencia práctica, en tanto producto de la etapa de reflexión, no han de ser vistas como estándares, sino como enunciados que expresan un razonamiento a partir de fines y medios que se juzgan justificados por el agente, en la etapa previa de reflexión (balance de razones). La tesis de la conexión puede ser presentada mediante la idea contraria: la identificación y conocimiento de un estándar moral implica que el agente debe seguir lo requerido por el estándar. Hacer esto, justifica el curso de acción elegido. Sin la identificación y conocimiento del estándar moral no es posible ni justificar ni elegir un curso de acción correcto.

25 Una versión más débil sería exigir que el agente proceda en forma paraconsistente, es decir, aceptando principios y razones y fines contradictorios en forma provisoria para después extraer todos los caminos posibles de justificación. A final de lo cual rechazará las razones, principios o fines inconsistentes con aquellos que haya elegido privilegiar. SERBENA (2005).
} 
La coherencia como ideal también puede, por otro lado, servir para dar sentido a una tercera noción de racional que se relaciona con el proceder que es consecuente con el final de la etapa de reflexión. Que el agente sea consecuente con la etapa previa de reflexión, a su vez, puede ocurrir en relación con el curso de acción que, de hecho, adopta, o bien, con la inferencia práctica que realiza. A este último respecto, cabe recordar que la finalización de la etapa de reflexión produce la identificación de fines, medios y la elección de un curso de acción. Se espera del agente que sea consecuente con la reflexión efectuada de la situación y que emplee el proceso de reflexión como insumo para la inferencia práctica: ${ }^{26}$ las premisas del silogismo práctico.

Al menos dos sentidos adicionales de racional pueden ser predicados de la etapa de reflexión en el razonamiento práctico. El primero está dado por el aspecto informativo y de verificación de las creencias. Racional es partir de información relevante, verdadera, respecto de la situación que pretende establecer si está justificada moralmente o no -p.ej. que compruebe la identidad del sujeto activo de la conducta que reprochará o que verifique si la proposición de que ha ocurrido cierto evento es verdadera-.

Finalmente, existe un sentido muy general de racional, por el que la reflexión es racional cuando se ofrecen razones: enunciados usados por el hablante para justificar los pasos y resultados del proceso de reflexión -frente a sí o frente a terceros-.

En síntesis, puede predicarse racionalidad de la etapa de reflexión del razonamiento práctico moral a partir de: (1) que el agente se conduzca tratando de dilucidar qué le exige la moral en la situación práctica; (2) el modo de proceder con la reflexión en forma consistente; (3) la consecución del curso de acción que adopta, o bien, con la inferencia práctica que realiza; (4) el acopio de la información pertinente y la verificación de sus creencias acerca del mundo y (5) el ofrecimiento de razones. Estos cinco sentidos sirven para evaluar los discursos de justificación en la etapa de reflexión de un razonamiento práctico moral.

Quedan excluidos de la visión del razonamiento práctico moral aquí defendido otros sentidos de racional. De entrada, el sentido que apela a la presencia de criterios compartidos de decisión o de procedimientos dotados de altos consensos para extraer conclusiones. Es decir, el sentido de racionalidad en tanto método compartido por una comunidad para identificar problemas y determinar qué cuenta como una solución. Se trata de las bases metodológicas comunes a los agentes morales que permitan la demostración, la verificación o el saber, en tanto creencia justificada en la verdad de enunciados.

\footnotetext{
${ }^{26}$ Lo que, por cierto, no implica que siempre que se realiza una reflexión se produce un discurso bajo la forma de una inferencia práctica. Puede ocurrir, en contextos muy simples o poco interesantes para el agente, que una vez concluida la reflexión simplemente actúe y que únicamente presente un silogismo práctico si se le pide justificación de su actuar.
} 
Algunas de tales bases metodológicas inexistentes en los razonamientos prácticos morales incluyen, entre otras: (1) la existencia de criterios comunes para identificar las propiedades relevantes en cada situación -el universo de propiedades relevantes-, (2) la semántica común entre los participantes de la discusión que determine la intensión de las propiedades relevantes -el sentido de los términos morales-, (3) el criterio para asignar la misma calificación moral a cada propiedad relevante -la valencia moral de la situación genérica-, (4) la existencia de criterios para determinar qué propiedades relevantes con su correlativa calificación moral -p.ej. "Mentir es moralmente indebido"- deben ser suscritas como fines que justifican la conducta de los agentes y, finalmente, lo más sobresaliente, (5) el método para decidir qué cuenta como un argumento concluyente a favor o en contra de cada una de las cuestiones antes enumeradas, en los escenarios de desacuerdo genuino. Sin la satisfacción de las bases metodológicas anteriores, la discusión acerca de la etapa de reflexión del razonamiento práctico moral carece de lo que podríamos llamar racionalidad de método. ${ }^{27}$ En ausencia de ello la evaluación del razonamiento práctico en forma pública, intersubjetiva y conspicua no está disponible. La consecuencia es un rasgo palpable del discurso moral: los desacuerdos parecen infranqueables y los acuerdos sobre un razonamiento concreto se reducen o bien a la convergencia fáctica de preferencias entre hablantes o bien a los mecanismos de persuasión (y disuasión) que se logran propinar entre sí los participantes.

Desde luego que los participantes de la discusión pueden intercambiar opiniones, los juicios de justificación que adoptan, la apreciación de coherencia entre los medios aceptados y los fines morales de los que parten, la compatibilidad de medios y su disponibilidad o costo. Pero el despliegue de este intercambio de opiniones no equivale a saldar las bases metodológicas comunes enunciadas anteriormente, ni implica la presencia de una racionalidad de método en el discurso moral.

Recapitulando, el carácter abierto y relativo al contexto del razonamiento práctico moral en su etapa de reflexión es compatible con cinco sentidos de racionalidad, que sirven para dotar de criterios de valoración acerca del balance de razones. El discurso de justificación moral carece, sin embargo, de la racionalidad de método.

Lo expuesto tiene la finalidad de abonar a una forma de caracterización del razonamiento jurídico como un razonamiento práctico, pero con exigencias de razón teórica y con algún grado de racionalidad de método que, por tanto, lo distinguen del razonamiento práctico moral.

\footnotetext{
27 Una idea semejante se puede extraer acerca del papel normativo que tiene el método en las ciencias naturales. LAUDAN (1990). También se puede encontrar esta misma idea en el papel que Guibourg le asigna al método en la elección de una ontología. GuiBOURG (2004).
} 


\section{Razonamiento jurídico}

El razonamiento jurídico es un discurso de justificación que ocurre en distintos ámbitos del derecho -tribunales, parlamentos, academia, opinión pública- y es realizado por distintos actores. Interesa ubicarnos únicamente en el razonamiento que hace un juez, en su encargo de juzgador y durante el curso de un caso, ${ }^{28}$ en el discurso que va dirigido a justificar la decisión final para el caso. A diferencia del razonamiento práctico moral, el jurídico posee exigencias de la razón teórica: conocimiento de normas y conocimiento de hechos. Esta exigencia es hecha en el contexto de los Estados modernos, ahí donde el diseño institucional establece que el juez debe fundar y motivar su fallo o sentencia. ${ }^{29}$

La primera exigencia es que el juez muestre que ciertas formulaciones de norma (disposiciones) sean las válidas y aplicables al caso (conocimiento de normas). El juez tiene que mostrar que está usando el derecho válido, aplicable al caso. Por ejemplo, de acuerdo con los criterios de identificación o en función del tipo de caso -p.ej. el código civil en la parte del contrato de compraventa que dice que el vendedor se hace cargo de los vicios ocultos que presente la cosa, para el caso de una controversia civil, la norma de competencia que dice que un juez de insolvencia, de quiebra y concurso, es competente para conocer de los contratos pendientes de ejecución-. Son exigencias de conocimiento, por lo que el juez debe ofrecer razones acerca de la creencia verdadera de que las disposiciones, válidas y aplicables al caso, son las que está empleando. Esto incluye tanto las normas que regulan el fondo de la controversia como las que regulan el proceso, la prueba, la competencia y la ejecución así como alguna interpretación hecha por un tribunal superior que vincula al juez. En algunos casos, el conjunto de disposiciones que puede elegir el juez para mostrar que sabe cuál es el derecho válido y aplicable al caso incluye más de una posibilidad, ${ }^{30}$ pero, en general, no es el caso. Por el contrario, en algunos contextos el universo de posibles disposiciones está acotado, incluso, por las partes: por ejemplo en el caso de los paneles arbitrales de

\footnotetext{
${ }^{28} \mathrm{El}$ análisis aquí presentado cambiaría para el caso de un legislador constituyente o una comisión redactora de un tratado internacional, por ejemplo. En cambio, para un legislador ordinario las exigencias de conocimiento jurídico que suelen imponer las constituciones modernas son parecidas mutatis mutandi a las del juez.

29 Es posible encontrar ejemplos históricos y diseños institucionales en lo que se realiza una decisión jurídica para producir una solución a un caso sin requerir de los agentes una justificación acerca del conocimiento del derecho (p.ej. un jurado de enjuiciamiento penal en los sistemas de common law). Lo mismo ocurre con la exigencia de conocer ciertos hechos: es posible encontrar ejemplos de diseños institucionales en donde el pronunciamiento del juez o la autoridad no incluyen justificar los hechos que conoce del caso. De tal modo que las exigencias de razón teórica que aquí caracterizan al razonamiento jurídico son relativas a un cierto contexto institucional.

${ }^{30}$ Por ejemplo cuando puede optar por emplear una norma de derecho nacional u otra de derecho internacional o en los casos en que concurre más de una norma para calificar la causa criminal o cuando la ley establece alternativas que puede declarar el juez al final de un juicio en la fase de sentencia.
} 
organismos supranacionales, en donde el insumo normativo le es configurado con antelación al juez. También puede suceder que por razón de alguna anomalía sintáctica, anfibología o vaguedad en los términos del texto o en los casos de lagunas normativas el conocimiento jurídico del juez sea indeterminado.

El tipo de exigencia y el grado de justificación que deba exhibir el juez acerca del conocimiento jurídico se regula de distintas maneras en cada ordenamiento jurídico, pero suele estar presente en todos.

El ámbito fáctico del caso también está regulado: se le exige al juez que posea conocimiento acerca de los hechos del caso (conocimiento de hechos). El universo de hechos a probar suele estar delimitado por el contexto legal del caso -lo que las partes discuten-, el juez debe mostrar qué hechos del caso dentro de ese contexto conoce. ${ }^{31}$ Asimismo el derecho regula la forma y modo en que el juez conoce los hechos: únicamente mediante la prueba ofrecida por las partes o mediante pruebas que el propio juez puede producir -o la prohibición de presentar algunos tipos de probanzas (inmorales) u obtenidas mediante tortura-. Pero también se regula el tipo de prueba, los tiempos y formas de presentarlas, el peso relativo que pueden tener -tasación- o cuándo debe tenerse por probado un hecho y con qué grado de certeza -el estándar de la prueba-. Dentro de este contexto se encuentran fines del legislador que a veces no van encaminados a averiguar la verdad de lo ocurrido sino a cumplir otros fines que suelen incidir en la tarea cognoscitiva del juez: aventajar a un grupo vulnerable -p.ej. presunciones a favor del trabajador- o disminuir la frecuencia en el uso de los recursos de la administración de justicia -p.ej. al incrementar los requisitos de procedencia de la prueba- o finalmente proteger ciertos valores - p.ej. la prueba obtenida por medios ilícitos-. A pesar de que existen diseños institucionales que inciden en la tarea epistémica del juez de conocer los hechos, ${ }^{32}$ el caso es que el derecho le exige al juez que tenga cierto conocimiento acerca de los hechos del caso y en esa medida es una tarea cognoscitiva, propia de la razón teórica.

La exigencia de incluir en las resoluciones que solucionan un caso la justificación epistémica de que el juez tiene conocimiento de normas y de hechos no afecta que el juez realiza, también, un balance de razones para justificar la elección de alternativas posibles -incluso si son alternativas acotadas por reglas institucionales-, y realiza una inferencia práctica. Es decir, el razonamiento jurídico se compone, también, de un razonamiento práctico.

La primera etapa del razonamiento práctico del juez será una de reflexión, un balance de razones cuya justificación es una relación entre un dominio de razones y un conjunto de acciones. Frente a alternativas de fuentes de derecho debe justificar cuál elige, lo mismo frente a posibles sentidos del texto normativo - o sentidos previamente atribuidos por otros órganos-, la concurrencia de más de

\footnotetext{
31 PARDO (2013); TARUFFO (2012).

32 LAUDAN (2006).
} 
un estándar normativo -regla, principio o directriz- lo obliga a justificar el arreglo y la forma en que los estándares concurren en el caso -sea que los armonice, jerarquice o pondere $y$, también, debe decidir la descripción que hará del caso individual para presentarlo como regulado por los estándares que ha elegido-. Otro tanto sucede con las operaciones de valoración de las pruebas: determinar qué datos de entre los aportados por las partes ha de elegir, la narración que hará de los hechos del caso, la relevancia de las pruebas para la discusión de los hechos del caso, el peso, las consecuencias que se extraen, ${ }^{33}$ justificar la afirmación de que el supuesto normativo está probado -o desaprobado-, decidir en qué grado las consecuencias que se siguen de los enunciados que asume como probados alcanzan para satisfacer el estándar de la prueba.

En una segunda fase se le exige al juez que muestre la inferencia práctica que produce en la fase de reflexión. Las premisas que asume y las consecuencias que deriva para el caso concreto. Es una exigencia también común en los estados de derecho que el juez articule sus razones a modo de un razonamiento, completo, claro, consistente, en el que se presentan las premisas y la conclusión con las que resuelve el asunto. Esta exigencia de revelar y comunicar el razonamiento no es siempre, ni las más de las veces, necesaria parar el razonamiento práctico moral.

Reconocer que el razonamiento jurídico tiene exigencias de razón teórica es un supuesto necesario para entender su naturaleza y para distinguirlo del razonamiento moral. ${ }^{34}$ No ya solamente porque en el razonamiento moral no hay conexión con la razón teórica, sino porque la conexión entre razón teórica y razonamiento jurídico provoca que podamos predicar de este algún grado mayor de racionalidad de método.

La racionalidad de método se predica en algún grado del razonamiento jurídico porque la comunidad jurídica no es una comunidad de método en el sentido de que posee consensos fuertes acerca de las operaciones de razonamiento, pruebas y justificación. Las prácticas jurídicas son diferentes a las prácticas de los ingenieros, biólogos, médicos o farmacéuticos. Sin embargo, ello no quiere decir que el razonamiento jurídico carezca de racionalidad de método, como es el caso del razonamiento moral.

\footnotetext{
33 ANDERSON, SCHUM y Twining (2005).

${ }^{34}$ Como se puede anticipar, aquí se sostiene una distinción de grado entre el razonamiento jurídico y el razonamiento moral. De un lado, porque hemos acotado la extensión de "razonamiento jurídico" al que realiza el juez que pone fin a una controversia. Del otro lado, porque el grado de razón teórica que presente un razonamiento jurídico está regulado institucionalmente. Así, se puede advertir que una regla institucional que no le exija al juez a justificar su conocimiento de normas y de hechos produciría un razonamiento jurídico equivalente a un razonamiento moral, en su estructura y modo de evaluación (p.ej. el razonamiento que puede exhibir un tribunal constitucional o un arbitraje de conciliación y paz internacional). Es decir, es posible que las reglas institucionales disminuyan el grado de exigencia en la justificación acerca de las creencias sobre normas y hechos del juzgador. Por lo que la distinción aquí propuesta es, inevitablemente, una de grado.
} 
El razonamiento jurídico, debido a su componente de razón teórica, exhibe algún grado de racionalidad de método. Ello es así porque, en general, los juristas encuentran en los actos legislativos y de tribunales fuentes comunes para identificar las propiedades relevantes generales -los supuestos jurídicos-. También sucede que la actividad de la dogmática genera un vocabulario técnico, muchas veces tomado por el legislador o los jueces que, en algunos casos, provee de una semántica convencional para dotar de sentido a las propiedades relevantes -sin perjuicio de los amplios debates de la dogmática acerca de las características que definen los términos-. Por otro lado, el estatus de las conductas es conocido por la práctica jurídica en los casos que regularmente son tratados como simples -sin perjuicio de que un caso simple siempre puede ser controvertido y de que existen casos que generan grandes desacuerdos acerca del estatus jurídico de una conducta-. Estos rasgos de las prácticas jurídicas hacen que el razonamiento jurídico tenga un mayor grado de racionalidad de método, para algunos casos de la práctica. Con todo, sin embargo, se adolece de un método para decidir qué cuenta como un argumento concluyente a favor o en contra de cada una de las cuestiones antes enumeradas, en los escenarios de desacuerdo: la identificación de las fuentes, la atribución de sentido de los textos, la semántica de los conceptos, el modo de solucionar los casos. No existe un consenso acerca de los criterios para decidir $-\mathrm{y}$ demostrar- las disputas argumentativas en el derecho. Y, a pesar de ello, el razonamiento jurídico puede ser sujeto a escrutinio y evaluación.

La evaluación del razonamiento jurídico admite cuatro dimensiones. La primera dimensión está dada por los criterios con los que juzgamos la formación de creencias y la justificación del conocimiento. Se evalúa si el juez cumplió con la exigencia institucional de tener el conocimiento jurídico y el conocimiento fáctico que se le exige por el derecho para el caso (razón teórica). De otro lado, en tanto razonamiento práctico, está la dimensión del criterio formal que evalúa si la conclusión se sigue de las premisas, en tanto inferencia. La tercera dimensión es la evaluación material de las premisas del razonamiento, relativas a la corrección de los fines y medios y el material que descansa en los cinco criterios de racionalidad que se tienen para la etapa de reflexión: (1) que el juez se conduzca tratando de dilucidar qué le exige el derecho en la situación práctica; (2) que el juez proceda en forma coherente con sus consideraciones para el caso; (3) que el resultado de sus consideraciones se vea reflejado en las premisas y en la conclusión de la inferencia práctica que realiza; (4) que muestre que tuvo toda la información pertinente y fiable para resolver el caso y (5) que ofrezca argumentos, razones.

El trazo que hemos hecho sobre la diferencia entre razonamiento jurídico y razonamiento práctico moral permite anunciar el análisis de dos cuestiones: 1) ¿En qué medida es el razonamiento jurídico una forma de razonamiento moral? y 2) ¿En qué medida la verdad juega un papel en el razonamiento jurídico? Dos interrogantes que deberán esperar en el tintero otra ocasión, pero cuyo esbozo de respuesta se puede inferir a partir de lo hasta aquí expuesto. 


\section{BIBLIOGRAFÍA}

* Alchourrón, Carlos E. y Martino, Antonio A. (1990): "Logic without truth", en Ratio Juris (Volume 3, No. 1).

* Anderson, Terence, Schum, David, y Twining, William (2005): Analysis of evidence (Cambridge University Press).

* Bratman, Michael (1987): Intention, Plans, and Practical Reason (Cambridge Massachusetts, Harvard University Press).

* BAYÓn, Juan Carlos (1991): La normatividad del derecho: Deber jurídico y razones para la acción (Madrid, Centro de estudios constitucionales).

* Caracciolo, Ricardo (2009): El derecho desde la filosofía (Madrid, Centro de Estudios Constitucionales).

* Dancy, Jonathan (2004): Ethics without Principles (Oxford, Oxford University Press).

* Duarte D’almeida, Luis (2013): “A Proof-Based Account of Legal Exceptions”, en Oxford Journal of Legal Studies (Vol. 33, No. 1).

* GeORge, Robert P. (2002): Para hacer mejores a los hombres. Libertades civiles y moralidad pública (Madrid, Ediciones Internacionales Universitarias).

* Greenawalt, Kent (2001): “Title VII and Religious Liberty", en Loyola University Chicago Law Journal (Loyola University Chicago School of Law).

* Guibourg, Ricardo A. (1999): Pensar en las normas (Buenos Aires, Eudeba).

* Guibourg, Ricardo A. (2004): La construcción del pensamiento: decisiones metodológicas (Buenos Aires, Colihue).

* Laudan, Larry (1990): Science and Relativism: some key controversies in the philosophy of science (Chicago, University of Chicago Press).

(2006) Truth, Error and Criminal Law: An Essay in Legal Epistemology (Nueva York, Cambridge University).

* LitTle, Margaret Olivia (2001): “On Knowing the 'Why': Particularism and Moral Theory", en The Hastings Center Report (Vol. 31, No. 4, The Hastings Center).

* Moore, S. Michael (2010): Causation and Responsability: an essay in Law, Morals, and Metaphysics (Oxford, Oxford University Press).

* Navarro, Pablo, Oronesu, Claudia, Rodríguez, Jorge L. y Sucar, Germán (2001): "La aplicabilidad de las normas jurídicas", en Analisi e diritto 2000. Ricerche di giurisprudenza analitica (Torino, Giappichelli).

* Pardo, Michael S. (2013): “The Nature and Purpose of Evidence Theory", en Vanderbilt Law Review (Vol. 66, No. 2).

* RaZ, J. (1990): Practical reason and norms (Princeton, Princeton University Press).

* Ryle, Gilbert (2002): Concept of mind (Chicago, University of Chicago Press).

* Serbena, Cesar Antonio (2005): "Colisiones de principios y lógica paraconsistente anotada", en Ideas y Derecho ( $\mathrm{N}^{\circ}$ ).

* Shafer-Landau, Russ (1997): "Moral Rules", en Ethics, University Chicago Press (Vol. 107, No. 4).

* Stanley, Jason y Williamson, Timothy (2001): "Knowing How", en Journal of Philosophy, Journal of Philosophy Inc. (Vol. 98, No. 8)

* Taruffo, Michelle (2012): Proceso y decisión. Lecciones mexicanas de Derecho Procesal (Madrid, Marcial Pons).

* Von Wright, Georg H. (1963): Norm and Action (Londres, Routledge and Kegan Paul). (1979) Explicación y comprensión (Madrid, Alianza Editorial).

* Williams, Bernard (1972): Morality: An introduction to ethics (Reino Unido, Cambridge University Press). 